\title{
A UTILIZAÇÃO DO PHOTOVOICE COMO ESTRATÉGIA PEDAGÓGICA
}

\author{
Maria Anabela Ferreira dos Santos ${ }^{1}$, Luísa Sotto-Mayor ${ }^{2}$, Madalena Oliveira ${ }^{3}$,Irene \\ Soares $^{4}$ e Isabel Serra ${ }^{5}$ \\ 1, 2, 3, 4, 5 Departamento de Enfermagem de Saúde Materna, Escola Superior de Enfermagem de Lisboa, Portugal. \\ 1'afsantos@esel.pt; ${ }^{2} \mathrm{ml}$ pinto@esel.pt; ${ }^{3}$ mmoliveira@esel.pt; ${ }^{4}$ imsoares@esel.pt; ${ }^{5 i s e r r a @ e s e l . p t ~}$
}

\begin{abstract}
Resumo. O presente artigo visa dar a conhecer o projeto em curso sobre aquisição de competências dos estudantes da Pós-Licenciatura e do Mestrado em Saúde Materna e Obstétrica e assim contribuir para a melhoria das práticas pedagógicas da unidade curricular Estágio com Relatório. Compreender o fenómeno do desenvolvimento de competências específicas do futuro Enfermeiro Especialista de Saúde Materna e Obstétrica. Estudo exploratório, qualitativo, utilizando o método Photovoice para a colheita e análise de dados (colheita de fotos e entrevistas) e posterior análise de conteúdo segundo Bardin com recurso ao WebQDA. Foram obtidas até ao momento, 29 fotos de 9 participantes, ainda em fase de análise. O método Photovoice permite aceder à perspetiva de desenvolvimento de competências dos estudantes, apreendendo a sua experiência do fenómeno através da captura de fotos significativas para o processo e da sua narrativa.
\end{abstract}

Palavras-chave: Photovoice; Competências; Enfermeira Obstetra.

\section{THE USE OF PHOTOVOICE AS A PEDAGOGICAL STRATEGY}

\begin{abstract}
The present work aims to make known the current project on the acquisition of competences of the students of the Post-Graduation and the Master in Maternal and Obstetric Health and thus contribute to the improvement of the pedagogical practices of Course Unit Internship with Report. To understand the phenomenon of the development of specific skills of the future Specialist Nurse in Maternal and Obstetric Health. Exploratory, qualitative study using the Photovoice method for the collection and analysis of data (collection of photos and interviews) and subsequent content analysis according to Bardin using WebQDA. So far, 29 photos of 9 participants were still being analyzed. The Photovoice method provides access to the perspective of students' skills development, learning their experience of the phenomenon through the capture of significant photos and their narrative.
\end{abstract}

Keywords: Photovoice; Skills; Obstetrical Nurse.

\section{INTRODUÇÃO}

Os Cursos de Pós-Licenciatura de Especialização em Enfermagem de Saúde Materna e Obstetrícia (CPLEESMO) e o Curso de Mestrado em Enfermagem de Saúde Materna e Obstetrícia (CMESMO) visam assegurar a aquisição e desenvolvimento de competências instrumentais, pessoais e sistémicas, nomeadamente as científicas, técnicas, humanas e culturais, necessárias ao Enfermeiro Especialista em Enfermagem de Saúde Materna e Obstetrícia. De acordo com o Plano de Estudos do Curso de Pós-Licenciatura de Especialização em Enfermagem de Saúde Materna e Obstétrica, apresentado à Ordem dos 
Enfermeiros ${ }^{1}$, o enfermeiro especialista nesta área, através do seu desempenho, contribui para a melhoria da qualidade das condições assistenciais à mulher ao longo do ciclo de vida, ao recém-nascido e família e, consequentemente, para a redução da morbilidade e mortalidade materna, perinatal e neonatal. Acreditamos que estes cursos têm contribuído para a melhoria da qualidade dos cuidados na área da saúde materna e consequentemente para a obtenção de ganhos em saúde.

Os estudantes do CPLEESMO e do CMESMO são detentores de uma formação inicial de $1^{\circ}$ ciclo, o Curso de Licenciatura em Enfermagem, sendo que aos primeiros são ainda exigidos dois anos de exercício de prática clínica, como enfermeiros de cuidados gerais, à data da candidatura ao referido curso. São por isso, estudantes adultos, muitos deles com vasta formação anterior, experiência profissional e maturidade pessoal e profissional. A frequência de um curso desta natureza representa um enorme investimento pessoal e profissional, não somente porque a maioria o acumula com o exercício profissional, como também com a vida familiar, que para a quase totalidade se encontra em fase de expansão com filhos pequenos. Por estes motivos, a conceção, planeamento e execução dos planos de estudo, assim como as metodologias adotadas têm em consideração a pedagogia de adultos, em particular o uso de metodologias interativas que envolvam os estudantes, tornando-os agentes ativos da sua aprendizagem e considerando-os o centro do processo de ensino-aprendizagem.

De acordo com o Guia Orientador, a UC Estágio com Relatório do CPLEESMO ${ }^{2}$ tem como finalidade: "desenvolver competências na prestação de cuidados especializados à mulher/RN/família, durante os diferentes estádios do trabalho de parto, puerpério e período neonatal" (ESEL; 2018, p.2).

\subsection{Utilização do Photovoice}

A análise qualitativa apresenta a visão interna dos participantes, a sua perspetiva, capturando a sua experiência do fenómeno através da narrativa. O Photovoice é um método cada vez mais utilizado na pesquisa qualitativa, por aumentar a possibilidade de captar as perceções e experiências dos participantes (Santos, 2018). As fotografias estimulam novos pensamentos e memórias despoletadas, mas não necessariamente contidas na imagem

\footnotetext{
${ }^{1}$ Proposta de Plano de Estudos do Curso de Pós-Licenciatura de Especialização em Enfermagem de Saúde Materna e Obstétrica, ESEL, 2018.

${ }^{2}$ Documento Orientador da Unidade Curricular Estágio com Relatório, ESEL, 2018.
} 
presente na fotografia, tornando o invisível visível e deste modo tornam possível aceder à compreensão de fenómenos ou de experiências. Para além disso, porque acrescentam ao sentido da audição o da visão, expandem a consciência sensorial e aumentam o processo reflexivo (Padgett, Smith, Derejko, Henwood, \& Tiderington, 2013). O conceito de Photovoice foi desenvolvido por Caroline Wang e Mary Ann Burris em 1997. Desde os primeiros estudos na década de noventa, o uso do Photovoice tem vindo a aumentar com participantes de ambos os sexos, de várias faixas etárias, de várias etnias e abordando um leque variado de temas na área da saúde e da justiça social, como por exemplo doenças, discriminação ou violência (Catalani \& Minkler, 2010). O termo Photovoice encerra em si mesmo a ideia central deste método - dar voz à experiência individual ou coletiva através das fotos (Santos, 2018). A participação ativa dos sujeitos, quer na tomada de decisão do que fotografar e quando, assim como na elicitação das fotos, estimula o pensamento crítico, promove a mudança e empodera-os (Liebenberg, 2018). O método Photovoice permite desocultar importante informação descritiva e é utilizado para responder a questões de investigação, que nortearam o presente estudo:

Quais são os momentos-chave para o estudante, promotores do desenvolvimento das competências específicas de Enfermeiro Especialista de Saúde Materna e Obstétrica, ao longo da Unidade Curricular Estágio com Relatório em contexto de Bloco de Partos?

Como consciencializam estes a aquisição e desenvolvimento destas competências? Que sentido atribuem a estas aprendizagens?

Que mudanças podemos implementar nas práticas pedagógicas utilizadas na UC "Estágio com Relatório" do CPLEESMO", que permitam potenciar o alcance das competências previstas para os futuros especialistas e a qualidade dos cuidados nesta área?

\section{METODOLOGIA}

O presente estudo encontra-se em fase de desenvolvimento. É um estudo qualitativo e exploratório que visa contribuir para a melhoria das práticas pedagógicas da Unidade Curricular (UC) Estágio com Relatório.

Tem como objetivo geral compreender o fenómeno do desenvolvimento de competências específicas do Enfermeiro Especialista de Saúde Materna e Obstétrica (EESMO), e como objetivos específicos: identificar os momentos-chave do desenvolvimento de competências específicas de Enfermagem de Saúde Materna e Obstétrica (ESMO) dos estudantes dos 
Cursos de Pós-Licenciatura e de Mestrado em estágio no bloco de partos; situar temporalmente a ocorrência desses momentos-chave; facilitar a tomada de consciência dos estudantes dos momentos-chave que contribuíram para o seu desenvolvimento de competências específicas de futuro ESMO; conhecer o significado que os estudantes atribuem aos momentos-chave do desenvolvimento de competências específicas do EESMO; consciencializar os estudantes do seu desenvolvimento de competências específicas do EESMO.

Os participantes são estudantes do $9^{\circ} \mathrm{CPLEESMO} \mathrm{e} \mathrm{do} 9^{\circ} \mathrm{CMESMO}$ da Escola Superior de Enfermagem de Lisboa a realizarem o Estágio com Relatório em contexto de Bloco de Partos. Pretende-se continuar a colheita de dados junto dos estudantes dos cursos subsequentes, até se obter saturação de dados.

Métodos de colheita e análise de dados:_Photovoice (colheita de fotos e entrevistas); utilização do método do Photovoice para a recolha de dados através de colheita de fotos e entrevistas subsequentes para elicitação do seu conteúdo; análise de conteúdo segundo Bardin. Google Forms para recolha dos dados sociodemográficos.

Foram introduzidas algumas alterações e adaptações à técnica do Photovoice (Santos, Lopes, \& Botelho, 2018) descrita por Wang e Burris (Wang \& Burris, 1997), devido à especificidade do estudo e dos participantes, sendo os procedimentos usados os seguintes: num primeiro momento os participantes foram informados do tema e objetivo do estudo e da utilização do Photovoice, solicitando-se-lhes que escolhessem momentos que considerassem ter contribuído, de alguma forma, para o seu desenvolvimento de competências específicas como futuro EESMO ao longo da unidade curricular Estágio com Relatório que estavam a realizar em contexto de Bloco de Partos. Pediu-se-Ihes também que fotografassem esses momentos e os descrevessem oralmente ou por escrito. Não foi imposto qualquer tipo de limitações quanto ao número ou material a ser fotografado. Apenas foi sugerido que fotografassem preferencialmente objetos ou lugares relacionados com o motivo que os levou a tirar essa fotografia, em vez de rostos, de modo a preservar a identidade e manter o anonimato dos participantes e dos clientes, bem como a dispensar o consentimento ético das instituições onde decorria o estágio. Foram obtidos a autorização e o consentimento de todos os estudantes que quiseram participar no estudo, para utilização das fotos e das narrativas, após Ihes ter sido explicado os objetivos do mesmo, a metodologia e a garantia do anonimato. Não 
foi necessário distribuir máquinas fotográficas ou instruir os participantes sobre o seu uso, porque todos possuíam telefones portáteis com câmaras acopladas que sabiam manejar.

Foram obtidas até ao momento, 29 fotos de 9 participantes.

Numa segunda fase serão realizadas entrevistas em profundidade em que serão discutidos, analisados e validados com o investigador as fotos capturadas e os temas identificados. Nesta fase, denominada photo elicitation (Harper, 2010; Plunkett, Leipert, \& Ray, 2013) , baseada na reflexão crítica através do diálogo e ação, será utilizada a técnica SHOWeD modificada, de forma a dar resposta aos objetivos. As perguntas baseadas no acrónimo SHOWeD (Wang, 2006) foram simplificadas e ajustadas aos objetivos do estudo, à especificidade e à realidade dos participantes, sendo a técnica SHOWeD usada apenas como guia orientador.

O acrónimo SHOWeD refere-se a questões a colocar aos participantes para os ajudar a falarem acerca das fotografias por eles capturadas, para as descreverem e analisarem criticamente. É composto pelas seguintes cinco perguntas ( Wang, 1999; Catalani \& Minkler, 2010; Padgett et al., 2013; Teti, Pichon, Kabel, Farnan, \& Binson, 2013): What do you See here? What is really Happening here? How does this relate to Our lives? Why does this situation, concern or strength exist? What can we Do about it?

Neste estudo, as perguntas a realizar serão as seguintes: O que o levou a tirar esta fotografia? Fale-me um pouco desta fotografia. De que trata esta fotografia? O que é mais importante nesta fotografia? O que pretende expressar/transmitir? Que significado teve para si este momento? Se tivesse que explicar esta fotografia, por telefone (ou a uma pessoa que não a pudesse ver) o que diria? Como a descreveria? De que modo este momento está relacionado com a aquisição de competências específicas do Enfermeiro Especialista de Saúde Materna e Obstétrica? Como é que esta imagem está relacionada com o seu desenvolvimento de competências no bloco de partos?

As entrevistas foram realizadas nos dias de orientação tutorial em que os estudantes se deslocaram à escola, ou nos serviços aquando das reuniões de orientação. As entrevistas foram gravadas em áudio, transcritas para um verbatim para posteriormente serem introduzidas e analisadas juntamente com as fotografias recolhidas, através do software WebQDA. 
Algumas entrevistas já foram realizadas, outras encontram-se em fase de transcrição e análise, estando prevista a continuidade deste procedimento. A cada estudante foi atribuído um número de código (E1, E2, etc.), bem como às entrevistas realizadas (ETV 1).

Os dados serão sujeitos a uma análise qualitativa, utilizando os procedimentos inerentes à análise de conteúdo segundo Bardin (2015). De acordo com o método desta autora, o verbatim das entrevistas será analisado da seguinte forma: uma primeira fase, de pré-análise, em que o material é submetido a uma leitura flutuante, de modo a que o investigador fique com uma ideia de todo o material produzido, permitindo a identificação do corpus da análise, ou seja, do material a ser sujeito a análise; na fase seguinte de análise, proceder-se-á ao recorte (escolha das unidades), enumeração (escolha das regras de contagem) e à classificação e agregação (escolha das categorias). Com este método de análise do conteúdo das entrevistas obtêm-se as categorias, subcategorias, as unidades de contexto, as unidades de registo e a frequência (Bardin, 2015).

Utilizaremos o software WebQDA para o tratamento dos dados qualitativos resultantes das entrevistas. Para o tratamento dos dados quantitativos relativos à caracterização sociodemográfica dos estudantes, será utilizado o programa SPSS.

\section{RESULTADOS}

Por se encontrar ainda em fase de desenvolvimento, ainda não existem resultados. Contudo, das 29 fotografias captadas pelos 9 estudantes no período compreendido entre 18 de Fevereiro e 5 de Julho de 2019, constatamos que alguns dos temas abordados pelas estudantes se centram nos seguintes aspetos que, posteriormente, após análise mais detalhada dos dados, são passíveis de vir a formar categorias: dificuldades relacionadas com deslocações e compatibilização de vida profissional e familiar, a importância da prática simulada em laboratório, o desenvolvimento de competências técnicas e instrumentais, em particular a interpretação do registo cardiotocográfico e a tomada de decisão sobre a necessidade de realização de episiorrafia, bem como a técnica inerente (figura 1), o desenvolvimento de competências teóricas e sua mobilização na prática clínica, a gestão de tempos e de espaços, a promoção da segurança (figura 2) e lidar com situações de perda e luto (figura 3). 


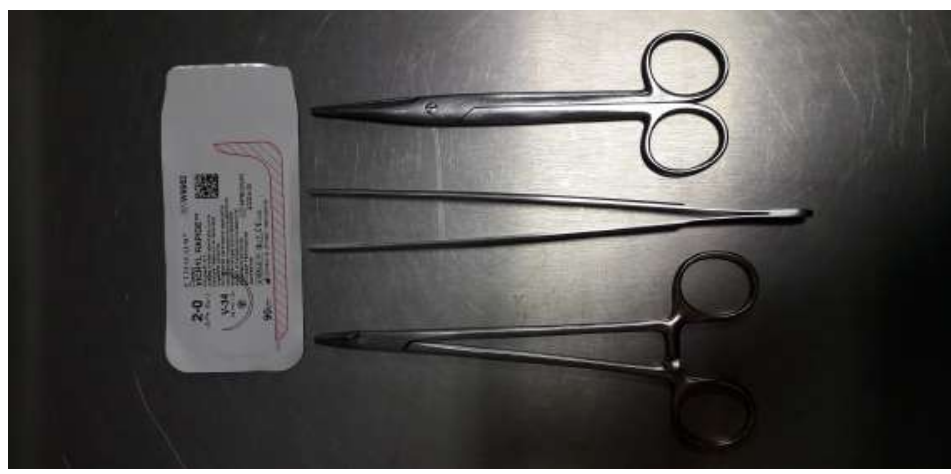

Figura 1. Exemplo de foto alusiva ao desenvolvimento de competências de reparação cirúrgica do períneo. (Fonte: foto recolhida pelo estudante E4)

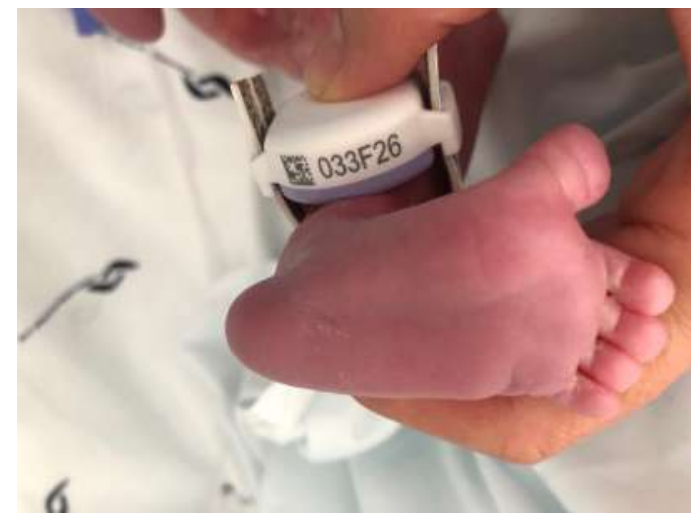

Figura 2. Exemplo de foto alusiva ao desenvolvimento de competências de promoção da segurança do recémnascido (Fonte: foto recolhida pelo estudante E6)

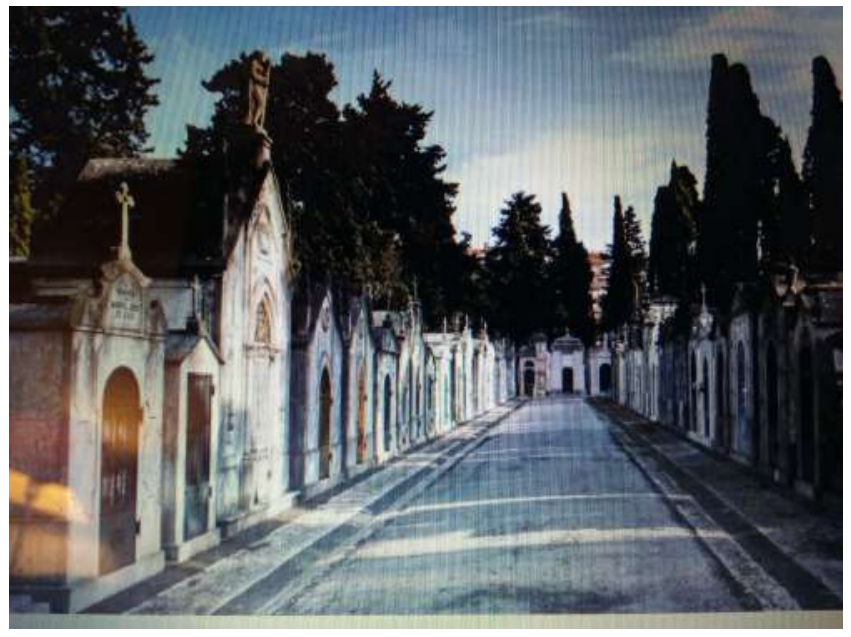

Figura 3 Exemplo de foto alusiva ao desenvolvimento de competências de promoção de coping com situações de perda e luto (Fonte: foto recolhida pelo estudante E5) 


\section{CONCLUSÕES}

Ao melhorar a compreensão do fenómeno do desenvolvimento das competências comuns e específicas dos estudantes, nomeadamente desocultando os momentos-chave deste processo, estamos a facilitar o processo pedagógico de desenvolvimento de competências por parte destes, a contribuir para a consciencialização das suas aquisições, a capacitá-los para a tomada de decisão e para uma atitude proativa de envolvimento no processo de ensino/aprendizagem. O método do Photovoice permitiu-nos aceder à perspetiva dos estudantes, apreendendo a sua experiência do fenómeno através da captura das fotos e das respetivas narrativas.

Alguns dados já obtidos, apontam para dificuldades relacionadas com a conciliação da vida profissional e familiar destes estudantes adultos. Também a gestão de tempos e de espaços visando a promoção da segurança (das clientes e a própria) e a prestação de cuidados de qualidade, atuando com base numa filosofia holística centrada na mulher, seus conviventes significativos e família. É salientada a importância do treino antecipado de situações de cuidados, mantendo, ou reforçando, a utilização das práticas simuladas em laboratório, as quais integram já a UC considerada, como experiência prévia ao ensino clínico. Algumas dificuldades relatadas no desenvolvimento de competências científicas, sua transferência e mobilização na prática clínica, exigem a continuidade de uma aposta numa prática baseada na melhor evidência, que permita aos estudantes tomarem decisões fundamentadas e afirmarem-se como elementos essenciais no seio das equipas multiprofissionais, assegurando altos padrões de qualidade nos cuidados prestados.

O desenvolvimento de competências técnicas e instrumentais, aparecem como uma preocupação major neste ensino clínico, em particular, a interpretação do registo cardiotocográfico, que permite despistar precocemente situações de risco materno-fetal, bem como a decisão sobre a necessidade de realização de episiorrafia e a execução da técnica inerente.

De salientar, ainda, a necessidade manifestada por alguns estudantes na consolidação de competências para lidar com situações de perda e luto, com as quais se confrontam na prática clínica. Estes resultados apontam para a necessidade de uma aposta continuada nas competências transversais (de comunicação e relação, científicas, éticas, técnicas...), a par da aquisição de novas competências específicas da área de especialidade considerada, 
reforçando a ideia de competência como algo flexível e sempre passível de ser melhorado, tendo a formação um papel preponderante nesta construção.

Este estudo, ainda em curso, permitirá aumentar a compreensão do fenómeno do desenvolvimento das competências dos estudantes a frequentar o CPLEESMO/CMESMO, fundamentando na evidência, possíveis mudanças processuais na UC "Estágio com Relatório", conducentes da melhoria das práticas e do processo pedagógico, como um todo. Tais mudanças, poderão situar-se ao nível do ajuste dos timings e/ou da inovação de ferramentas pedagógicas que permitam agilizar e potenciar o alcance das competências previstas para o perfil de competências do futuro Enfermeiro Especialista em Saúde Materna Obstétrica.

\section{REFERÊNCIAS}

Bardin, L. (2015). Análise de conteúdo. (5 ed). Lisboa: Edições70.

Catalani, C., \& Minkler, M. (2010). Photovoice: A Review of the Literature in Health and Public Health. Health Education \& Behavior, 37(3), 424-451. http://doi.org/10.1177/1090198109342084.

Diretiva n. ${ }^{\circ}$ 80/155/CEE (1980). que tem por objetivo a coordenação das disposições legislativas, regulamentares e administrativas relativas ao acesso às atividades de parteira e ao seu exercício. Diretiva do Conselho. Jornal Oficial, nº L 033 (de 11/02/1980). 0008 - 0012 de 21 de janeiro de 1980.

Escola Superior de Enfermagem de Lisboa (2018). Documento Orientador da Unidade Curricular. Estágio com Relatório: ESEL, Lisboa.

Harper, D. (2010). Talking about pictures: A case for photo elicitation. Visual Studies, 17(1), $13-26$. http://doi.org/10.1080/1472586022013734.

International Confederation of Midwives (2018). Essential competencies for basic midwifery practice: 2018 Update (Netherlands): ICM.

Le Boterf, G. (2017). Agir en professionnel compétent et avec éthique. Éthique Publique, 19 (1). [on line] : http://journals.openedition.org/ethiquepublique/2934. DOI : https://doi.org/10.4000/ethiquepublique.2934.

Liebenberg, L. (2018). Thinking Critically About Photovoice : Achieving. Empowerment and Social Change, 17, 19. http://doi.org/10.1177/1609406918757631.

Padgett, D. K., Smith, B. T., Derejko, K.-S., Henwood, B. F., \& Tiderington, E. (2013). A picture is worth . . ? Photo elicitation interviewing with formerly homeless adults. Qualitative Health Research, 23(11), 1435-44. http://doi.org/10.1177/1049732313507752.

Plunkett, R., Leipert, B. D., \& Ray, S. L. (2013). Unspoken phenomena: Using the photovoice method to enrich phenomenological inquiry. Nursing Inquiry, 20(2), 156-164. http://doi.org/10.1111/j.14401800.2012.00594.x.

Regulamento n. 140 (2019). Ordem dos Enfermeiros. Regulamento das Competências Comuns do Enfermeiro Especialista. Diário da República, $2 .^{a}$ série (N. ${ }^{\circ} 26$ de 6 de fevereiro de 2019). 
Regulamento n. ${ }^{\circ} 391$ (2019). Ordem dos Enfermeiros. Regulamento das Competências Específicas do Enfermeiro Especialista em Enfermagem de Saúde Materna e Obstétrica. Diário da República, 2. ${ }^{a}$ série (N. ${ }^{\circ} 85$ de 3 de maio de 2019).

Santos, M. A. F. (2018). O processo de metamorfose da mulher acima dos trinta e cinco anos em mãe : uma teoria específica da situação. Universidade de Lisboa.

Santos, M. A., Lopes, M. A., \& Botelho, M. A. (2018). Photovoice as a method of data collection in the study of motherhood over the age of 35: the power of images. In A. P. Costa, L. P. Reis, F. N. Souza, \& A. Moreira (Eds.), Computer Supported Qualitative Research, Advances in Intelligent Systems and Computing (pp. 316326). Springer. http://doi.org/10.1007/978-3-319-61121-1.

Teti, M., Pichon, L., Kabel, A., Farnan, R., \& Binson, D. (2013). Taking pictures to take control: Photovoice as a tool to facilitate empowerment among poor and racial/ethnic minority women with HIV. Journal of the Association of Nurses in AIDS Care, 24(6), 539-553. http://doi.org/10.1016/j.jana.2013.05.001.

Wang, C. (2006). Youth Participation in Photovoice as a Strategy for Community Change. Journal of Community Practice, 14(1-2), 147-161. http://doi.org/10.1300/J125v14n01_09 147.

Wang, C., \& Burris, M. A. (1997). Photovoice: concept, methodology, and use for participatory needs assessment. Health Education \& Behavior, 24(3), 369-387. http://doi.org/10.1177/109019819702400309.

Wang, C. C. (1999). Photovoice: A participatory action research strategy applied to women's health. Journal of Women's Health. http://doi.org/10.1089/jwh.1999.8.185 